\title{
Non-equilibrium dynamics: quantum systems and foundations of quantum mechanics
}

\author{
Václav Špička ${ }^{1, a}$, Peter D. Keefe ${ }^{2}$, and Theo M. Nieuwenhuizen ${ }^{3}$ \\ ${ }^{1}$ Institute of Physics, Czech Academy of Sciences, Prague, Czech Republic \\ ${ }^{2}$ College of Engineering and Science, University of Detroit Mercy, Detroit, USA \\ ${ }^{3}$ Institute for Theoretical Physics, University of Amsterdam, Amsterdam, The \\ Netherlands
}

Received 4 February 2019

Published online 5 March 2019

\begin{abstract}
This text presents a brief overview of the recent development of topics addressed by the original papers of this volume related to nonequilibrium phenomena in various (especially mesoscopic) systems and the foundations of quantum physics. A selection of relevant literature is included.
\end{abstract}

\section{Introduction}

The volume summarizes advances in the understanding of the behavior of quantum systems out of equilibrium, together with related themes of the foundation of quantum physics and quantum thermodynamics. The original contributions are from top scientists of these fields (and their collaborators) who participated in the conference Frontiers of Quantum and Mesoscopic Thermodynamics (FQMT'17), which was organized by the Institute of Physics of the Czech Academy of Sciences and was held in Prague in July 2017 as a follow up to the six previous FQMT conferences (https://fqmt.fzu.cz/) [1-5].

The volume contains several mini-review papers and tutorials which provide readers an excellent orientation into the state-of-the-art of the following topics: Nonequilibrium statistical physics and quantum transport, Quantum thermodynamics and molecular engines, Foundations of quantum physics, Disordered and quantum many body systems, superfluidity, and superconductivity, and Biophysics and chemical physics. These reviews are accompanied by original research papers dealing with more detailed aspects of these fields. We believe this set of papers provides useful insights into recent developments in the above mentioned fields and at the same time the volume documents important relations between related topics.

Further development of all these fields is needed to deal with an increasing requirement for more detailed understanding and use of such phenomena as quantum correlations, entanglement and their dynamics; decoherence and dissipation; light-matter interactions; behavior of closed and open quantum systems far from equilibrium; equilibration and thermalization of systems; roles of initial and boundary conditions; influences of environment, reservoirs and external fields on the

a e-mail: spicka@fzu.cz 
time evolution of systems; quantum to classical transitions; dynamics of quantum phase transitions; and topological states of systems. The above mentioned phenomena, related problems and challenges occur in many fields of physics, astrophysics, chemistry, and biology.

As for systems, which enable study of various related questions, mesoscopic systems are especially suitable for this purpose due to their vast variety of structures and parameters. Various systems, of natural and artificial origin, can exhibit mesoscopic features depending on inner parameters of those systems and interactions with their environment. Typical mesoscopic systems can be of nanoscale size, composed from atoms (molecules). Nanoscale structures include not only very small physical structures, but also structures occurring in living cells, as for example complex molecules, proteins and molecular motors. At the same time, nanoscale technologies enable the preparation of well-defined artificial structures composed of between a few to hundreds of atoms (molecules) to create an enormous diversity of systems with well-defined inner parameters which can be influenced by external fields. These structures can be studied by methods of condensed matter physics and quantum optics in such detail that affords a deeper understanding of quantum physics, as represented by quantum interference, entanglement, the uncertainty principle, quantum measurement and what is often termed "non-locality". Of particular interest are carbon allotropes, quantum wires and dots, microcavities, single molecule nanomagnets, molecular motors and active gels, various structures in living cells, as well as specific arrangements featuring cold atoms and molecules which can exhibit macroscopic quantum effects and which can be used for testing methods of quantum many-body theory.

Recent advances in technologies have led to enormous improvements of measurement, imaging and observation techniques at microscopic, mesoscopic and macroscopic scales. At the same time, various methods allow investigation into not only equilibrium features, but also time evolution of classical and quantum systems (which are in general far from equilibrium) at different time scales. This increasing ability to study subtle details of the dynamics of systems yields new versions of old questions and creates new challenges in many fields of physics.

A good understanding of the time evolution of both classical and quantum systems is essential for an explanation of many observations and experiments of contemporary physics. Observed systems must often be treated as non-equilibrium, open systems in which their behavior is influenced not only by their inner parameters, but also by properties of their environment and time dependent external fields. The theory of non-equilibrium behavior of quantum many-body systems is, however, far from complete. There are lasting and extremely important problems related to modern technologies, including questions of irreversible behavior of real systems in comparison with reversible microscopic laws, emergence of classical macroscopic behavior from microscopic quantum behavior, charge (electron), spin and heat transport, limits to "phenomenological" thermodynamic descriptions, and the problem of how to describe properly open quantum systems far from equilibrium, especially in the case of strong interaction between a small system and reservoirs.

Another challenging problem is stochastic behavior of systems caused either by innate features of the systems or by noise related to the fact that the studied systems are open. Studies of quantum and temperature fluctuations, as well as quantum noise, dephasing and dissipation create an essential part of the research in this direction. Recently, various versions of non-equilibrium fluctuation and fluctuationdissipation theorems for quantum systems have been discussed. These studies are of key importance since the fluctuations, dissipation and noise are closely related to the performance and the reliability of both artificially created nano-devices as well as natural "engines", as are for example molecular motors in cells. 
This theme brings us to the field of biophysics. Non-equilibrium processes and the system's environment play a decisive role in the behavior of small structures of living organisms and there are many important questions to be answered before we fully understand the laws which govern the performance of the nanoscopic structures that are essential for life. In this regard, it appears one of the necessary conditions for the proper performance of cells is that their dynamics be based on far from equilibrium states and related nonlinear, non-equilibrium transport.

Behavior of molecular motors and the field of biomimetics are associated with more general considerations related to thermodynamics and the use of various mesoscopic structures. Among the central themes of classical thermodynamics are the concepts of "temperature", "system", "reservoir", and "engine". Due to the quantum aspects of mesoscopic (molecular) systems, it is necessary to deal with quantum thermodynamics to discuss possible quantum pumps, heat engines and refrigerators. The task of quantum thermodynamics is to provide a good "phenomenological" frame for the "macroscopic" description of open mesoscopic systems coming from more detailed studies of non-equilibrium quantum statistical physics of open systems and the foundations of quantum mechanics. The central question is: under which conditions will the thermodynamic behavior still manifest in various small systems.

In general, the above mentioned problems arise in dissipation, dephasing and decoherence processes, and, on a very basic level, the foundations of quantum mechanics and related theories of quantum measurement. A better knowledge and insight into the foundations of quantum physics is essential for a proper formulation of the fundamental laws of physics with regard to Bell inequalities and quantum gravity. It is also essential for developing a suitable description of small quantum systems and their applications. This applies particularly to quantum optics and the physics of quantum information and computing, where questions of quantum interference, entanglement and decoherence processes, together with knowledge of time scales governing the dynamics of the studied systems, are essential and mutually beneficial.

The contributions to this topical issue have been grouped in five sections, as indicated in the contents of the volume. Additional details of recent developments regarding the subjects of individual sections can be found in the short list of books and review articles (ordered mostly by years of publication):

- Non-equilibrium statistical physics and quantum transport: from references [6-65],

- Quantum thermodynamics and molecular engines: from references [66-88],

- Foundations of quantum physics: from references [89-149],

- Disordered and quantum many body systems, superfluidity, and superconductivity: from references [150-187],

- Biophysics and chemical physics: from references [188-227].

We note that only recent books and review articles are referred to. Our choice is, of course, rather subjective and far from complete. Nevertheless, we trust the reader has been provided a basic orientation to the fields and topics contained in this volume.

We are indebted to Peter Hänggi, Sabine Lehr, Nicolas Puyaubreau, and Sandrine Karpe for all their support, efforts and professional work throughout the preparation of this special issue. We would also like to thank all other members of EPJ ST for their help. 


\section{References}

1. Th.M. Nieuwenhuizen, P.D. Keefe, V. Špička (Eds.), Proceedings of the International Conference on Frontiers of Quantum and Mesoscopic Thermodynamics (FQMT04), Physica E 29, 1 (2005)

2. Th.M. Nieuwenhuizen, P.D. Keefe, V. Špička (Eds.), Proceedings of the International Conference on Frontiers of Quantum and Mesoscopic Thermodynamics (FQMT08), Physica E 42, 207 (2010)

3. Th.M. Nieuwenhuizen, P.D. Keefe, V. Špička (Eds.), FQMT11: Topical Issue - International Conference on Frontiers of Quantum and Mesoscopic Thermodynamics, Physica Scripta T151, 010301 (2012) , 17, 83, 96, 158.

4. Th.M. Nieuwenhuizen, P.D. Keefe, V. Špička (Eds.), FQMT13:Topical Issue - International Conference on Frontiers of Quantum and Mesoscopic Thermodynamics, Physica Scripta T165, 010301 (2015)

5. P.D. Keefe, Th.M. Nieuwenhuizen, V. Špička (Eds.), Topical Issue - International Conference on Frontiers of Quantum and Mesoscopic Thermodynamics (FQMT15), Fortschritte der Physik (Progress of Physics) 65, 1720206 (2017) 1. Non-equilibrium statistical physics and quantum transport

6. R. Balian, in From Microphysics to Macrophysics (Springer-Verlag, Berlin, 1992), Vol. II

7. C.W. Gardiner, Handbook of Stochastic Methods for Physics, Chemistry and the Natural Sciences, 2nd edn. (Springer, Berlin, 1985, 1997)

8. R. Dean Astumian, F. Moss (Eds.), The construction role of noise in fluctuation driven transport and stochastic resonance, Chaos 8, 3 (1998)

9. T. Dittrich, P. Hänggi, G.-L. Ingold, B. Kramer, G. Schön, W. Zwerger, Quantum Transport and Dissipation (Wiley, 1998)

10. M. Grifoni, P. Hänggi, Driven Quantum Tunnelling, Phys. Rep. 304, 229 (1998)

11. L. Gammaitoni, P. Hänggi, P. Jung, F. Marchesoni, Stochastic Resonance, Rev. Mod. Phys. 70, 223 (1998)

12. C.W. Gardiner, P. Zoller, Quantum Noise, A Handbook of Markovian and Nonmarkovian Quantum Stochastic Methods with Applications to Quantum Optics (Springer, Berlin, 2000)

13. Stochastic processes in physics, chemistry and biology, edited by J.A. Freund, T. Poschel (Springer, Berlin, 2000)

14. G. Lang, U. Weiss, Nonlinear quantum transport and current noise, Ann. Phys. (Leipzig) 9, 804 (2000)

15. R. Zwanzig, Nonequilibrium Statistical Mechanics (Oxford University Press, 2001)

16. H.D. Zeh, The Physical Basis of the Direction of Time, 4th edn. (Springer, Berlin, 2001)

17. V. Pechakus, U. Weiss (Eds.), Quantum Dynamics of Open Systems, Chem. Phys. 268, $1(2001)$

18. H.P. Breuer, F. Petruccione, The Theory of Open Quantum Systems (Oxford, New York, 2002)

19. R.L. Liboff, Kinetic Theory, Classical, Quantum and Relativistic Descriptions, 3rd edn. (Prentice Hall, London, 1990, Springer, 2003)

20. H.J. Carmichael, Statistical Methods in Quantum Optics 1: Master Equations and Fokker-Planck Equations (Springer, 1999)

21. H.J. Carmichael, Statistical Methods in Quantum Optics 2: Non-Classical Fields (Springer, 2008)

22. H. Haug, A.P. Jauho, Quantum Kinetics in Transport and Optics of Semiconductors, 2nd edn. (Springer, 2008)

23. G.E. Crooks, On the Jarzynski relation for dissipative quantum mechanics, J. Stat. Phys. 2008, P10023 (2008)

24. M. Griffoni, E. Paladino (Eds.), Focus on Quantum Dissipation in Unconventional Environments, New J. Phys. 10, 115003 (2008)

25. U. Weiss, Quantum Dissipative Systems, 3rd edn. (World Scientific, 2008) 
26. Y.V. Nazarov, Y.M. Blanter, Quantum transport (Cambridge University Press, 2009)

27. F. Ritort, Fluctuations in open systems, Physics 2, 43 (2009)

28. M. Esposito, U. Harbola, S. Mukamel, Nonequilibrium fluctuations, fluctuation theorems, and counting statistics in quantum systems, Rev. Mod. Phys. 81, 1665 (2009)

29. A.K. Paul, S. Adhikari, M. Baer, A treatise on the interaction of molecular systems with short-pulsed highly-intense external fields, Phys. Rep. 496, 79 (2010)

30. J.C. Cuevas, E. Sheer, Molecular Electronics: An Introduction to Theory and Experiment (World Scientific, 2010)

31. C. Jarzynski, Equalities and Inequalities: Irreversibility and the Second Law of Thermodynamics at the Nanoscale, Annu. Rev. Con. Matt. Phys. 2, 329 (2011)

32. E. Akkermans, G. Montambaux, Mesoscopic Physics of Electrons and Photons (Cambridge University Press, 2011)

33. A. Kamenev, Field Theory of Non-Equilibrium Systems (Cambridge University Press, Cambridge, 2011)

34. N.A. Zimbovskayaa, M.R. Pederson, Electron transport through molecular junctions, Phys. Rep. 509, 1 (2011)

35. A. Polkovnikov, K. Sengupta, A. Silva, M. Vengalattore, Colloquium: Nonequilibrium dynamics of closed interacting quantum systems, Rev. Mod. Phys. 83, 863 (2011)

36. R. Friedrich, J. Peinke, M. Sahimi, M. Reza Rahimi Tabar, Approaching complexity by stochastic methods: From biological systems to turbulence, Phys. Rep. 506, 87 (2011)

37. C. Jarzynski, Single-molecule experiments: Out of equilibrium, Nat. Phys. 7, 591 (2011)

38. Y. Dubi, M. Di Ventra, Colloquium: Heat flow and thermoelectricity in atomic and molecular junctions, Rev. Mod. Phys. 83, 131 (2011)

39. S. Datta, Lessons from Nanoelectronics (World Scientific, Cambridge, Singapore, 2012)

40. H. Gea, M. Qian, H. Qian, Stochastic theory of nonequilibrium steady states. Part II: Applications in chemical biophysics, Phys. Rep. 510, 87 (2012)

41. Nonequilibrium Statistical Physics of Small Systems: Fluctuation Relations and Beyond, edited by R. Klages, W. Just, C. Jarzynski (Wiley-VCH, Weinheim, 2012)

42. R. Tindjong, I. Kaufman, D.G. Luchinsky, P.V. McClintock, I. Khovanov, R.S. Eisenberg, Non-equilibrium Stochastic Dynamics of Open Ion Channels, Nonlin. Phenom. Comp. Syst. 16, 146 (2013)

43. K. Balzer, M. Bonitz, Nonequilibrium Green's Functions Approach to Inhomogenious Systems (Springer, Berlin, 2013)

44. G. Stefanucci, R. van Leeuwen, Nonequilibrium Many-Body Theory of Quantum Systems: A Modern Introduction (Cambridge University Press, Cambridge, 2013)

45. V. Špička, B. Velický, A. Kalvová, Electron systems out of equilibrium: Nonequilibrium Green's functions approach, Int. J. Mod. Phys. B 28, 1430013 (2014)

46. S. Denisov, S. Flach, P. Hänggi, Tunable transport with broken space-time symmetries, Phys. Rep. 538, 77 (2014)

47. E. Paladino, Y.M. Galperin, G. Falci, B.L. Altshuler, 1/f noise: Implications for solidstate quantum information, Rev. Mod. Phys. 86, 361 (2014)

48. C. Di Castro, R. Raimondi, Statistical Mechanics and Applications in Condensed Matter (Cambridge University Press, 2015)

49. E. Dieterich, J. Camunas-Soler, M. Ribezzi-Crivellari, U. Seifert, F. Ritort, Single-molecule measurement of the effective temperature in non-equilibrium steady states, Nat. Phys. 11, 971 (2015)

50. A. Klopper, Topics in non-equilibrium physics, Nat. Phys. Insight 11, 103 (2015)

51. M. Bonitz, Quantum Kinetic Theory, 2nd edn. (Springer, 2016)

52. L. Wang, N. Li, P. Hänggi, Simulation of heat transport in low-dimensional oscillator systems, in Thermal transport in low dimensions: From statistical physics to nanoscale heat transfer, edited by S. Lepri (Springer-Verlag, Berlin, Heidelberg, New York), Lecture Notes in Physics 921, 239 (2016)

53. C. Verdozzi, A. Wacker, C.O. Almbladh, M. Bonitz (Eds.), Progress in Nonequilibrium Green's Functions VI, J. Phys.: Conf. Ser. 696, 011001 (2016) 
54. H.-P. Breuer, E.-M. Laine, J. Piilo, B. Vacchini, Colloquium: Non-Markovian dynamics in open quantum systems, Rev. Mod. Phys. 88, 021002 (2016)

55. D.K. Morr, Crossover from quantum to classical transport, Contemp. Phys. 57, 19 (2016)

56. L.M. Sieberer, M. Buchhold, S. Diehl, Keldysh Field Theory for Driven Open Quantum Systems, Rep. Prog. Phys. 79, 096001 (2016)

57. M. Bonitz, Quantum Kinetic Theory, 2nd edn. (Springer, 2017)

58. V. Špička, B. Velický, A. Kalvová, Non-equilibrium dynamics of open systems and fluctuation-dissipation theorems, Fortschr. Phys. 65, 1700032 (2017)

59. I. de Vega, D. Alonso, Dynamics of non-Markovian open quantum systems, Rev. Mod. Phys. 89, 015001 (2017)

60. A. Ghosh, Nanoelectronics: A Molecular View (World Scientific, 2017)

61. S. Rotter, S. Gigan, Light fields in complex media: Mesoscopic scattering meets wave control, Rev. Mod. Phys. 89, 015005 (2017)

62. N.J. Morgenstern Horing Quantum Statistical Field Theory: An Introduction to Schwinger's Variational Method with Green's Function Nanoapplications, Graphene and Superconductivity (Oxford University Press, 2017)

63. V. Baltz, A. Manchon, M. Tsoi, T. Moriyama, T. Ono, Y. Tserkovnyak, Antiferromagnetic spintronics, Rev. Mod. Phys. 90, 015005 (2018)

64. T. Dornheim, S. Groth, M. Bonitz, The uniform electron gas at warm dense matter conditions, Phys. Rep. 744, 1 (2018)

65. F. Sebastian Bergeret, M. de Lardizabal, M. Silaev, P. Virtanen, T.T. Heikkila, Colloquium: Nonequilibrium effects in superconductors with a spin-splitting field, Rev. Mod. Phys. 90, 041001 (2018) 2. Quantum thermodynamics and molecular engines

66. M.B. Plenio, V. Vedral, Teleportation, entanglement and thermodynamics in the quantum world, Contemp. Phys. 39, 431 (1998)

67. F. Ritort, Work Fluctuations and Transient Violations of the Second Law: Perspectives in Theory and Experiments, Semin. Poincare 2, 63 (2003)

68. J. Gemmer, M. Michel, G. Mahler, Quantum Thermodynamics, Emergence of Thermodynamic Behavior within Composite Quantum Systems, Lecture Notes in Physics 657 (Springer, Berlin, 2004)

69. A.E. Allahverdyan, R. Balian, T.M. Nieuwenhuizen, Quantum thermodynamics: thermodynamics at the nanoscale, J. Mod. Opt. 51, 2703 (2004)

70. A.E. Allahverdyan, T.M. Nieuwenhuizen, Explanation of the Gibbs paradox within the framework of quantum thermodynamics, Phys. Rev. E 73, 066119 (2006)

71. M. Horodecki, Reversible path to thermodynamics, Nat. Phys. 4, 833 (2008)

72. F.G.S.L. Brandao, M.B. Plenio, Entanglement theory and the second law of thermodynamics, Nat. Phys. 4, 873 (2008)

73. P. Hänggi, F. Marchesoni, Artificial Brownian motors: Controlling transport on the nanoscale, Rev. Mod. Phys. 81, 387 (2009)

74. M. Campisi, P. Hänggi, P. Talkner, Colloquium: Quantum fluctuation relations: Foundations and applications, Rev. Mod. Phys. 83, 771 (2011)

75. U. Seifert, Stochastic thermodynamics, fluctuation theorems, and molecular machines, Rep. Prog. Phys. 75, 126001 (2012)

76. D. Bercioux, R. Egger, P. Hänggi, M. Thorwart (Eds.), Focus on nonequilibrium fluctuation relations: from classical to quantum, New J. Phys. 17, 020201 (2015)

77. B.P. Venkatesh, G. Watanabe, P. Talkner, Quantum fluctuation theorems and power measurements, New J. Phys. 17, 075018 (2015)

78. M. Campisi, J. Pekola, R. Fazio, Nonequilibrium fluctuations in quantum heat engines: Theory, example, and possible solid state experiments, New J. Phys. 17, 035012 (2015)

79. J.P. Pekola, Towards quantum thermodynamics in electronic circuits, Nat. Phys. 11, $118(2015)$

80. G. Gour, M.P. Muller, V. Narasimhachar, R.W. Spekkens, N.Y. Halpern, The resource theory of informational nonequilibrium in thermodynamics, Phys. Rep. 583, 1 (2015) 
81. J. Goold, M. Huber, A. Riera, L. dia del Rio, P. Skrzypczyk, The role of quantum information in thermodynamics? A topical review, J. Phys. A: Math. Theor. 49, 143001 (2016)

82. S. Vinjanampathy, J. Anders, Quantum thermodynamics, Contemp. Phys. 57, 545 (2016)

83. S. Alipour, F. Benatti, F. Bakhshinezhad, M. Afsary, S. Marcantoni, A.T. Rezakhani, Correlations in quantum thermodynamics: Heat, work, and entropy production, Sci. Rep. 6, 35568 (2016)

84. G. Katz, R. Kosloff, Quantum thermodynamics in strong coupling: Heat transport and refrigeration, Entropy 18, 186 (2016)

85. J. Anders, M. Esposito (Eds.), Focus on Quantum Thermodynamics, New J. Phys. 19, $010201(2017)$

86. F. Berger, S. Klumpp, R. Lipowsky, Force-dependent unbinding rate of molecular motors from stationary optical trap data, arXiv:1806.07865v1 [physics.bio-ph] (2018)

87. R. Alicki, R. Kosloff, Introduction to Quantum Thermodynamics: History and Prospects, arXiv:1801.08314v2 [quant-ph] (2018)

88. S. Popescu, A. Belén Sainz, A.J. Short, A. Winter, Quantum reference frames and their applications to thermodynamics, Phil. Trans. R. Soc. A 376, 20180111 (2018)

\section{Foundations of quantum physics}

89. A. Peres, Quantum Theory: Concepts and Methods (Kluwer Academic Publishers, 1995)

90. B. d'Espagnat, Veiled Reality (Addison-Wesley, 1995)

91. D.M. Greenberger, A. Zeilinger (Eds.), Fundamental Problems in Quantum Theory (Proceedings of a conference held in honor of J.A. Wheeler), Ann. N.Y. Acad. Sci. 755, 1 (1955)

92. L. de la Pena, A.M. Cetto, The Quantum Dice - An Introduction to Stochastic Electrodynamics (Kluwer Academic Publishers, 1996)

93. D. Bouwmeester, J.W. Pan, K. Mattle, M. Eibl, H. Weinfirter, A. Zeilinger, Experimental Quantum Teleportation, Nature 390, 575 (1997)

94. C.J. Isham, Lectures on Quantum Theory, Mathematical and Structural Foundations (Imperial College Press, 1995, 1997)

95. L. Hardy, Spooky action at a distance in quantum mechanics, Cont. Phys. 39, 416 (1998)

96. E.B. Karlsson, E. Brandes (Eds.), Modern Studies of Basic Quantum Concepts and Phenomena, Phys. Scr. 76, 4 (1998)

97. A. Zeilinger, Experiment and the Foundations of Quantum Physics, Rev. Mod. Phys. 71, S288 (1999)

98. A. Zeilinger, A foundational principle for quantum mechanics, Found. Phys. 29, 631 (1999)

99. B.G. Englert, M.O. Scully, H. Walter, Quantum erasure in double-slit interferometers with which-way detectors, Am. J. Phys. 67, 325 (1999)

100. H.A. Bethe, Quantum Theory, Rev. Mod. Phys. 71, S1 (1999)

101. J.T. Cushing, The interpretation of quantum mechanicsthrough 1935, Ann. Phys. (Leipzig) 9, 9392000

102. J. Ellis, D. Amati (Eds.), Quantum Reflections (Cambridge University Press, 2000)

103. G. Auletta, Foundations and Interpretation of Quantum Mechanics: In the Light of a Critical Historical Analysis of the Problems and of a Synthesis of the Results (World Scientific, 2000)

104. F. Laloe, Do we really understand quantum mechanics? Strange correlations, paradoxes and theorems, Am. J. Phys. 69, 655 (2001)

105. K.A. Milton, The Casimir Effect, Physical Manifestation of Zero-Point Energy (World Scientific, 2001)

106. W.M. De Muynck Foundations of Quantum Mechanics, An Empiricists Approach (Kluwer, Dordrecht, 2002) 
107. D. Giulini, E. Joos, C. Kiefer, J. Kupsch, I.O. Stamatescu (Eds.), Decoherence and the Appearance of a Classical World in Quantum Theory, 2nd edn. (Springer, Berlin, 1996, 2003)

108. M. Schlosshauer, Decoherence, the Measurement Problem, and Interpretations of Quantum Mechanics, Rev. Mod. Phys. 76, 1267 (2004)

109. A. Elitzur, S. Dolev, N. Kolenda (Eds.), Quo Vadis Quantum Mechanics? (Springer, Berlin, 2005)

110. G. Greenstein, A.G. Zajonc, The Quantum Challenge: Modern Research on the Foundations of Quantum Mechanics (Jones and Bartlett Publishers, Sudbury, 2006)

111. S. Popescu, A.J. Short, A. Winter, Entanglement and the foundations of statistical mechanics, Nat. Phys. 2, 754 (2006)

112. S. Haroche, J.-M. Raimond, Exploring the Quantum: Atoms, Cavities, and Photons (Oxford University Press, Oxford, 2006)

113. M. Schlosshauer, Decoherence and the Quantum-to-Classical Transition (Springer, Berlin, 2007)

114. L. Amico, R. Fazio, A. Osterloh, V. Vedral, Entanglement in many-body systems, Rev. Mod. Phys. 80, 517 (2008)

115. A.J. Leggett, Realism and the physical world, Rep. Prog. Phys. 71, 022001 (2008)

116. G. Auletta, M. Fortunato, G. Parisi, Quantum Mechanics (Cambridge University Press, 2009)

117. P.C. Hohenberg, Colloquium: An introduction to consistent quantum theory, Rev. Mod. Phys. 82, 2835 (2010)

118. M. Aspelmeyer, Measured measurement, Nat. Phys. 5, 11 (2009)

119. R. Horodecki, P. Horodecki, M. Horodecki, K. Horodecki, Quantum Entanglement, Rev. Mod. Phys. 81, 865 (2009)

120. GJ09 G. Jaeger, Entanglement, Information, and the Interpretation of quantum mechanics (Springer, 2009)

121. Ch. Ross, Dynamics of entanglement, Nat. Phys. 4, 97 (2008)

122. L. Maccone, V. Giovannetti, Quantum metrology: Beauty and the noisy beast, Nat. Phys. 7, 376 (2011)

123. Y. Sergeev, Quantum turbulence: Energy dissipation in extreme cold, Nat. Phys. 7, $451(2011)$

124. J.T. Barreiro, Quantum physics: Environmental effects controlled, Nat. Phys. 7, 927 (2011)

125. P.D. Nation, J.R. Johansson, M.P. Blencowe, F. Nori, Colloquium: Stimulating uncertainty: Amplifying the quantum vacuum with superconducting circuits, Rev. Mod. Phys. 84, 1 (2012)

126. K. Hornberger, S. Gerlich, P. Haslinger, S. Nimmrichter, M. Arndt, Colloquium: Quantum interference of clusters and molecules, Rev. Mod. Phys. 84, 157 (2012)

127. A.G. Kofman, S. Ashhab, F. Nori, Nonperturbative theory of weak pre- and postselected measurements, Phys. Rep. 520, 43 (2012)

128. J.I. Cirac, P. Zoller, Goals and opportunities in quantum simulation, Nat. Phys. 8, 264 (2012)

129. I. Bloch, J. Dalibard, S. Nascimbene, Quantum simulations with ultracold quantum gases, Nat. Phys. 8, 267 (2012)

130. A.E. Allahverdyan, R. Balian, Th.M. Nieuwenhuizen, Understanding quantum measurement from the solution of dynamical models, Phys. Rep. 525, 1 (2013)

131. Y.V. Nazarov, J. Danon, Advanced Quantum Mechanics: A Practical Guide (Cambridge University Press, 2013)

132. I. Georgescu (Ed.), Foundations of quantum mechanics, Nat. Phys. Insight 10, 253 (2014)

133. J. Dressel, M. Malik, F.M. Miatto, A.N. Jaordan, R.B. Boyd, Colloqium: Understanding quantum weak values: Basics and applications, Rev. Mod. Phys. 86, 307 (2014)

134. L. de la Pena, A.M. Cetto, A. Valdes-Fernandez, The Emerging Quantum: The Physics Behind Quantum Mechanics (Springer, 2015) 
135. G. Brennen, E. Giacobino, Ch. Simon (Eds.), Focus on Quantum Memory, New J. Phys. 17, 050201 (2015)

136. G. 't Hooft The Cellular Automaton Interpretation of Quantum Mechanics, in Fundamental Theories of Physics (Springer, 2016), Vol. 185

137. F. Zurek, Particle physics and condensed matter: the saga continues, Phys. Scr. T168, $014003(2016)$

138. J. Cotler, F. Zurek, Entangled histories, Phys. Scr. T168, 014004 (2016)

139. W.P. Bowen, G.J. Milburn, Quantum Optomechanics (Taylor and Francis Group, 2016)

140. A.E. Allahverdyan, R. Balian, Th.M. Nieuwenhuizen, A sub-ensemble theory of ideal quantum measurement processes, Ann. Phys. 376, 324 (2017)

141. T. Norsen, Foundations of Quantum Mechanics: An Exploration of the Physical Meaning of Quantum Theory (Springer, 2017)

142. D.E. Bruschi, A. Xuereb, Mechano-optics: an optomechanical quantum simulator, New J. Phys. 20, 06500 (2018)

143. O. Di Stefano, A.F. Kockum, A. Ridolfo, S. Savasta, F. Nori, Photodetection probability in quantum systems with arbitrarily strong light-matter interaction, Sci. Rep. 8, $17825(2018)$

144. N. Gisin, F. Fröwis, From quantum foundations to applications and back, Phil. Trans. R. Soc. A 376, 20170326 (2018)

145. W.G. Unruh, Locality and quantum mechanics, Phil. Trans. R. Soc. A 376, 20170320 (2018)

146. C. Agnesi, F. Vedovato, M. Schiavon, D. Dequal, L. Calderaro, M. Tomasin, D.G. Marangon, A. Stanco, V. Luceri, G. Bianco, G. Vallone, P. Villoresi, Exploring the boundaries of quantum mechanics: advances in satellite quantum communications, Phil. Trans. R. Soc. A 376, 20170461 (2018)

147. W.H. Zurek, Quantum reversibility is relative, or does a quantum measurement reset initial conditions? Phil. Trans. R. Soc. A 376, 20170315 (2018)

148. G. Adesso, R. Lo Franco, V. Parigi (Eds.), Foundations of quantum mechanics and their impact on contemporary society, Phil. Trans. R. Soc. A 376, 2123 (2018)

149. A. Acín, I. Bloch, H. Buhrman, T. Calarco, C. Eichler, J. Eisert, D. Esteve, N. Gisin, S.J. Glaser, F. Jelezko, S. Kuhr, M. Lewenstein, M.F. Riedel, P.O. Schmidt, R. Thew, A. Wallraff, I. Walmsley, F.K. Wilhelm, The quantum technologies roadmap: a European community view, New J. Phys. 20, 080201 (2018)

\section{Disordered and quantum many body systems, superfluidity, and superconductivity}

150. G.D. Mahan, Many Particle Physics (Plenum Press, New York, 1990)

151. L.P. Pitaevskii, S. Stringari, Bose-Einstein Condensation (Oxford University Press, Oxford, 2003)

152. H. Bruus, K. Flensberg, Many Body Quantum Theory in Condensed Matter Physics (Oxford University Press, Oxford, 2004)

153. C. Cohen-Tannoudji, J. Dupont-Roc, G. Grynberg, Photons and Atoms: Introduction to Quantum Electrodynamics (Wiley-VCH, Wenheim, 2004)

154. J.F. Annett, Superconductivity, Superfluidity and Condensates (Oxford University Press, Oxford, 2004)

155. Ch.J. Foot, Atomic Physics (Oxford University Press, Oxford, 2005)

156. A.J. Leggett, Quantum Liquids (Oxford University Press, Oxford, 2006)

157. A. Posazhennikova, Colloquium: Weakly interacting, dilute Bose gases in 2D, Rev. Mod. Phys. 78, 1111 (2006)

158. I. Bloch, P. Zoller (Eds.), Focus on Cold Atoms in Optical Lattices, New J. Phys. 8 (2006), https://iopscience.iop.org/article/10.1088/1367-2630/8/8/E02/meta

159. D.M. Greenberg, N. Erez, M.O. Scully, A.A. Svidzinsky, M.S. Zubairy, Planck, Photon Statistics, and Bose-Einstein Condensation, in Progress in Optics, edited by E. Wolf (Elsevier, Amsterdam, 2007), p. 275

160. R. Blatt, D. Wineland, Entangled states of trapped atomic ions, Nature 453, 1008 (2008) 
161. J. Clarke, F.K. Wilhelm, Superconducting quantum bits, Nature 453, 1031 (2008)

162. R. Hanson, D.D. Awschalom, Coherent manipulation of single spins in semiconductors, Nature 453, 1031 (2008)

163. I. Bloch, J. Dallibard, W. Zwerger, Many-body physics with ultracold atoms, Rev. Mod. Phys. 80, 885 (2008)

164. S. Giorgini, L.P. Pitaevskii, S. Stringari, Theory of ultracold atomic Fermi gases, Rev. Mod. Phys. 80, 1215 (2008)

165. I. Bloch, Quantum coherence and entanglement with ultracold atoms in optical lattices, Nature 453, 1016 (2008)

166. I. Bloch, Quantum gases in optical lattices, Science 319, 1202 (2008)

167. S. Diehl, A. Micheli, A. Kantian, B. Kraus, H.P. Buchler, P. Zoller, Quantum states and phases in driven open quantum systems with cold atoms, Nat. Phys. 4, 878 (2008)

168. C.J. Pethick, H. Smith, Bose-Einstein Condensation in Dilute Gases, 2nd edn. (Cambridge University Press, 2008)

169. A. Amo, D. Sanvitto, F.P. Laussy, D. Ballarini, E. del Valle, M.D. Martin, A. Lemaitre, J. Bloch, D.N. Krizhanovskii, M.S. Skolnick, C. Tejedor, L. Vina, Collective fluid dynamics of a polariton condensate in a semiconductor microcavity, Nature 457, 291 (2009)

170. C.J. Griffin, T. Nikuni, E. Zaremba, Bose Condensed Gases at Finite Temperatures (Cambridge University Press, 2009)

171. K. Dieckmann, Quantum gases: An insulating mix, Nat. Phys. 7, 593 (2011)

172. L.N. Cooper, D. Feldman (Eds.), BCS: 50 years (World Scientific, 2011)

173. W. Vassen, C. Cohen-Tannoudji, M. Leduc, D. Boiron, Ch.I. Westbrook, A. Truscott, K. Baldwin, G. Birkl, P. Cancio, M. Trippenbach, Cold and trapped metastable noble gases, Rev. Mod. Phys. 84, 175 (2012)

174. A. Imambekov, T.L. Schmidt, L.I. Glazman, One-dimensional quantum liquids: Beyond the Luttinger liquid paradigm, Rev. Mod. Phys. 84, 1253 (2012)

175. Z.-L. Xiang, S. Ashab, J.Q. You, F. Nori, Hybrid quantum circuits: Superconducting circuits interacting with other quantum systems, Rev. Mod. Phys. 85, 623 (2013)

176. M. Inguscio, L. Fallani, Atomic Physics: Precise Measurements and Ultracold Matter (Oxford, 2013)

177. C. Mudry, Lecture notes on field theory in condensed matter physics (World Scientific, 2014)

178. F. Nori, R.W Simmonds, J. You (Eds.), Focus on Quantum Microwave Field Effects in Superconducting Circuits, New J. Phys. (2014), https://iopscience.iop. org/journal/1367-2630/page/Focus\%20on\%20Quantum\%20Microwave\%20Field\% 20Effects\%20in\%20Superconducting\%20Circuits

179. P. Coleman, Introduction to Many-Body Physics (Cambridge University Press, Cambridge, 2015)

180. M. Brando, D. Belitz, F.M. Grosche, T.R. Kirkpatrick, Metallic quantum ferromagnets, Rev. Mod. Phys. 88, 025006 (2016)

181. X.-G. Wen, Colloquium: Zoo of quantum-topological phases of matter, Rev. Mod. Phys. 89, $041004(2017)$

182. C. Gardiner, P. Zoller, The Quantum World of Ultra-Cold Atoms and Light Book I: Foundations of Quantum Optics (World Scientific, 2014)

183. C. Gardiner, P. Zoller, The Quantum World of Ultra-Cold Atoms and Light Book II: The Physics of Quantum-Optical Devices (World Scientific, 2015)

184. C. Gardiner, P. Zoller, The Quantum World of Ultra-Cold Atoms and Light Book III: Ultra-cold Atoms (World Scientific, 2017)

185. E.C. Marino, Quantum Field Theory Approach to Condensed Matter Physics (Cambridge University Press, 2017)

186. F. Fröwis, P. Sekatski, W. Dur, N. Gisin, N. Sangouard, Macroscopic quantum states: Measures, fragility, and implementations, Rev. Mod. Phys. 90, 025004 (2018)

187. D.E. Chang, J.S. Douglas, A. González-Tudela, C.-L. Hung, H.J. Kimble, Colloquium: Quantum matter built from nanoscopic lattices of atoms and photons, Rev. Mod. Phys. 90, $031002(2018)$ 


\section{Biophysics and chemical physics}

188. B. Alberts, D. Bray, J. Lewis, M. Raff, K. Roberts, J.D. Watson, Molecular Biology of the Cell, 3rd edn. (Garland Publishing, Inc., 1994)

189. M.P. Murphy, L.A.J. O'Neill (Eds.), What is life? The Next Fifty Years (Cambridge University Press, Cambridge, 1995)

190. T.F. Weiss, Cellular Biophysics, Vol. 1 Transport (MIT Press, Cambridge, Massachusets, 1996)

191. T.F. Weiss, Cellular Biophysics, Vol. 2 Electrical Properties (MIT Press, Cambridge, Massachusets, 1996)

192. J.J. Hopfield, Dynamics, Computation, and Neurobiology, in Critical Problems in Physics, edited by V.L. Fitch, D.R. Marlow, M.A.E. Dementi (Princeton University Press, 1997), p. 29

193. A.S. Perelson, G. Weisbuch, Immunology for physicists, Rev. Mod. Phys. 69, 1219 (1997)

194. B.D. Hames, N.M. Hooper, J.D. Houghton, Instant Notes in Biochemistry (Bios Scientific Publishers, 1997)

195. H. Frauenfelder, G. Hummer, R. Garcia (Eds.), Biological Physics (American Institute of Physics, 1999)

196. M. Daune, Molecular Biophysics (Oxford University Press, Oxford, 1999)

197. H. Frauenfelder, P.G. Wolynes, R.H. Austin, Biological Physics, in More in Heaven and Earth, A Celebration of Physics at the Millenium, edited by B. Bederson (Springer, 1999), p. 707

198. J. McFadden, Quantum Evolution: Life in the Multiverse (Flamingo, London, 2000)

199. V. Sayakanit, L. Matson, H. Frauenfelder (Eds.), Biological Physics 2000 (World Scientific, Singapore, 2001)

200. S. Kauffman, Investigations (Oxford University Press, New York, 2000)

201. P. Nelson, Biological Physics (W.H. Freeman and Company, 2004)

202. D. Abbott, Quantum Aspects of Life (World Scientific, Singapore, 2007)

203. F. Ritort, Nonequilibrium fluctuations in small systems: From physics to biology, cond-mat 0705.0455 (2007)

204. F. J"ulicher, J. Käs (Eds.), Focus on Physics of the Cytoskeleton, New J. Phys. 10, 1367 (2007)

205. H.J. Briegel, S. Popescu, Entanglement and intra-molecular cooling in biological systems? A quantum thermodynamic perspective, arXiv:quant-phys/0806.4552 (2008)

206. M.B. Plenio, S.F. Huelga, Dephasing-assisted transport: quantum networks and biomolecules, New J. Phys. 10, 113019 (2008)

207. H. Frauenfelder, Proteins, supercooled liquids, and glasses: A micro-review, Physica E 42, $662(2010)$

208. A. Mossa, J.M. Huguet, F. Ritort, Investigating the thermodynamics of small biosystems with optical tweezers, Physica E 42, 666 (2010)

209. K.D. Dorfman, DNA electrophoresis in microfabricated devices, Rev. Mod. Phys. 82, $2903(2010)$

210. I. Baccarelli, I. Bald, F.A. Gianturco, E. Illenberger, J. Kopyrad, Electron-induced damage of DNA and its components: Experiments and theoretical models, Phys. Rep. 508, 1 (2011)

211. V.P. Zhdanov, Kinetic models of gene expression including non-coding RNAs, Phys. Rep. 500, 1 (2011)

212. G.D. Scholes, Quantum biology: Coherence in photosynthesis, Nat. Phys. 7, 448 (2011)

213. R. Philips, J. Kondev, J. Theriot, H. Garcia, Physical Biology of the Cell, 2nd edn. (Garland Science, 2013)

214. N. Lambert, Y.-N. Chen, Y.-C. Cheng, C.-M. Li, G.-Y. Chen, F. Nori, Quantum biology, Nat. Phys. 9, 10 (2013)

215. K.E. Dorfman, D.V. Voronine, S. Mukamel, M.O. Scully, Photosynthetic reaction center as a quantum heat engine, PNAS 110, 2746 (2013)

216. M. Mohseni, Y. Omar, G.S. Engel, M.B. Plenio (Eds.), Quantum Effects in Biology, 1st edn. (Cambridge University Press, 2014) 
217. H.B. Bohidar, Fundamentals of Polymer Physics and Molecular Biophysics (Cambridge University Press, 2015)

218. B. Alberts, A.D. Johnson, J. Lewis, D. Morgan, H. Raff, P. Walter, Molecular Biology of the Cell, 6th edn. (Garland Publishing, Inc., 2015)

219. J.F. Allemand, P. Desbiolles (Eds.), Physics and biology: from molecules to life (Singapore, World Scientific, 2015)

220. K. Kroy, E. Frey, Focus on soft mesoscopics: physics for biology at a mesoscopic scale, New J. Phys. 17, 110203 (2015)

221. E. Betzig, Nobel Lecture: Single molecule, cells, and super-resolution oprics, Rev. Mod. Phys. 87, 1153 (2015)

222. P.R. ten Wolde Putting Bounds on Biochemical Noise, Physics 9, 16 (2016)

223. R. Cortini, M. Barbi, B.R. Caré, C. Lavelle, A. Lesne, J. Mozziconacci, J.-M. Victor, The physics of epigenetics, Rev. Mod. Phys. 88, 025002 (2016)

224. Z. Zeravcic, V.N. Manoharan, M.P. Brenner, Colloquium: Toward living matter with colloidal particles, Rev. Mod. Phys. 89, 031001 (2017)

225. S. Klumpp, A. Siryaporn, S. van Teeffelen (Eds.), Focus on Bacterial Mechanics, New J. Phys. (2018), https://iopscience.iop.org/journal/1367-2630/page/Focus\% 20on\%20Bacterial\%20Mechanics

226. H. Miller, Z. Zhou, J. Shepherd, A.J.M. Wollman, M.C. Leake, Single-molecule techniques in biophysics: a review of the progress in methods and applications, Rep. Prog. Phys. 81, 024601 (2018)

227. S.J. Jang, B. Mennucci, Delocalized excitons in natural light-harvesting complexes, Rev. Mod. Phys. 90, 035003 (2018) 